\title{
Integration of an Adaptive Infotainment System in a Vehicle and Validation in Real Driving Scenarios
}

\author{
Miguel Angel Galarza, ${ }^{1}$ Teresa Bayona, ${ }^{2}$ and Josep Paradells ${ }^{3}$ \\ ${ }^{1}$ Universitat Politècnica de Catalunya, Barcelona, Spain \\ ${ }^{2}$ SEAT, S. A., Barcelona, Spain \\ ${ }^{3}$ Universitat Politècnica de Catalunya and Fundació I2CAT, Barcelona, Spain \\ Correspondence should be addressed to Miguel Angel Galarza; galarza.miguel@gmail.com
}

Received 15 September 2016; Revised 20 December 2016; Accepted 16 January 2017; Published 15 February 2017

Academic Editor: Radu Danescu

Copyright (C) 2017 Miguel Angel Galarza et al. This is an open access article distributed under the Creative Commons Attribution License, which permits unrestricted use, distribution, and reproduction in any medium, provided the original work is properly cited.

\begin{abstract}
More services, functionalities, and interfaces are increasingly being incorporated into current vehicles and may overload the driver capacity to perform primary driving tasks adequately. For this reason, a strategy for easing driver interaction with the infotainment system must be defined, and a good balance between road safety and driver experience must also be achieved. An adaptive Human Machine Interface (HMI) that manages the presentation of information and restricts drivers' interaction in accordance with the driving complexity was designed and evaluated. For this purpose, the driving complexity value employed as a reference was computed by a predictive model, and the adaptive interface was designed following a set of proposed HMI principles. The system was validated performing acceptance and usability tests in real driving scenarios. Results showed the system performs well in real driving scenarios. Also, positive feedbacks were received from participants endorsing the benefits of integrating this kind of system as regards driving experience and road safety.
\end{abstract}

\section{Introduction}

Modern society has become very dynamic, and the way in which people interact with services and obtain information from the environment has greatly changed over recent years. Individuals are becoming accustomed to having access to these new services very frequently and in any location, which has led to a certain dependency on technology and the need to be always "online." Regardless of the different attitudes regarding the benefits, drawbacks and origin of this phenomenon, it is clear that business models must evolve and adapt their strategies to cope with these demands.

Vehicle manufacturers, aware of the phenomena, are starting to provide new connectivity capabilities and more powerful hardware that enable users to maintain the connectivity while driving. In the very near future, In-vehicle Information Systems (IVIS) installed in vehicles will provide all types of services related to entertainment; vehicle maintenance, driving assistance, environmental alerts and social networks, as well as broad range of features aimed at improving driver experience [1]. This large array of services and functionalities is known as infotainment systems (a portmanteau word formed from "information" and "entertainment").

The integration of these new services and functionalities within a vehicle must be carefully planned. In a driving scenario, the design of the Human Machine Interface (HMI) must guarantee not only a good driving experience, but also, and more importantly, assuring a low level of distraction that otherwise may adversely affect road safety.

The approach currently adopted in vehicles consists in restricting the interaction with complex services when the vehicle is moving. These limitations affect user experience and undermine the real purpose of the application. On the contrary, supposing a driving scenario where multiple interfaces and functionalities are available to the driver, the driver may well reach a point of overload and distraction, especially when the road scenario is complicated. This leads us to the question of how much of this information the driver 
is able to process before it becomes a safety risk or degrades the driver experience.

The purpose of this study is to propose and validate some design principles useful for creating an adaptive HMI system based on driving complexity. The system was tested in real driving scenarios and participants were asked to give their opinions regarding safety and driving experience. An evaluation in real roads permits to receive a valuable feedback since the system is evaluated in the scenario for which it was designed. The development of the predictor for estimating driving complexity has been tested and validated in an independent study and is beyond the scope of this present study.

The paper is structured as follows: Some background is first presented regarding people's attention in a driving context; reference is made to some previously developed adaptive systems as well as to some HMI design principles. Next is presented the procedure followed in order to evaluate and implement the system by means of an acceptance test in a real driving scenario. Finally, the results are discussed and the conclusions presented.

\section{Background}

2.1. Driver Attention and Awareness. The activity of driving is quite complex and requires a high level of attention, since small distractions or errors occurring at high speeds may have a great impact on road safety. In 1970, McKnight and Adams suggested that approximately 40 primary driving tasks exist that a driver must learn in order to perform the driving activity successfully [2]. The additional introduction of secondary tasks interferes with these primary tasks and may compromise passengers safety.

In 2015, 26,300 deaths were registered in the EU due to driving accidents, while in the USA in 2014 32,675 fatalities were recorded for the same reason $[3,4]$. According to a report by the U.S. Department of Transportation, $10 \%$ of fatal crashes and $18 \%$ of injury crashes in 2014 were reported as a consequence of distractions [5]. In another study, it was found that $38 \%$ of the sample claimed to have been in an accident or had come close to being in a collision while driving when using a mobile phone or another kind of technology [6].

According to the International Road Transport Union, the main cause of driving accidents is human error, which accounts for up to $85.2 \%$ of the total number of accidents recorded [7]. These errors are usually associated with a driver overconfidence, misjudgement or inattention. Many drivers feel capable of performing nonprimary driving tasks when driving and a small misjudgement or a momentary lack of attention could seriously compromise road safety. In [8] the significant effect of performing secondary tasks is highlighted while driving on driver workload; in this study several visual patterns were measured and analysed in a driving simulator. Results showed the great impact of secondary tasks on visual workload.

Similarly, the accurate performance of driving tasks depends on the driving scenario; roads are heterogeneous and may be urban, rural, freeway-type, roads with bends or with different traffic density, to say nothing of the presence of animals, pedestrians and cyclists $[9,10]$. Driving performance may also be affected by environmental circumstances such as rain, fog or different conditions of light and visibility [11]. Finally, the skills and ability required to execute drivingrelated tasks vary from person to person and they consist of a series of cognitive, physical, and age features that characterize each individual [12].

In a diverse driving scenario, it is not always possible for drivers to process all the information, and in a consequence only a part is selected for interpretation; this process of discrimination is referred to as attention. One of the most comprehensive models of attention was proposed in 1984 by Wickens [13]. He defined a three-dimensional model composed of three stages: resources, modality, and the processing code. In this model two tasks interfere with each other only if they pass through the same channel. For example, the task of identifying a signal and expressing its meaning will interfere with other tasks such as detecting a pedestrian on the road and announcing his or her presence.

2.2. Previous Works regarding Vehicular Adaptive HMIs. This section presents an outline of the most relevant works and research dealing with adaptive HMIs. One of the most complete programs devoted to this topic is the "SAfety VEhicles using adaptive Interface Technologies" program (SAVE-IT), a 5-year research program sponsored by the "National Highway Traffic Safety Administration" (NHTSA) and administered by "The National Transportation Systems Center" (Volpe) [14]. This program was aimed at reducing distraction-related crashes by the implementation of adaptive countermeasures by monitoring the driver state.

The study consisted in the evaluation of an adaptive system based on the driver state using a driving simulator. This adaptive system was mainly focused on the integration of visual, auditory and haptic warnings in response to expected threats or hazards. It also provided a basic restriction mechanism that locked out certain features such as incoming calls by disabling some buttons available in the interface.

Further significant research work in the area of adaptive interfaces was the "Adaptive Integrated Driver-vehicle interfacE” (AIDE). This European program involved 31 partners and consisted of several research phases aimed at estimating driver workload and the definition of guidelines for regulating the presentation of information [15].

Some other research programs devoted to the improvement of road safety consisted of projects as "Generic Intelligent Driver Support System” (GIDS) (reported in [16]), "Application of Real-time Intelligent Aid for Driving and Navigation Enhancement" (ARIADNE) [17] and the "Communication Multimedia UNit Inside CAR" (COMUNICAR) [15]. The main premise of these projects was the definition of schedulers that managed a timely presentation of information depending on the scenario complexity or driver workload. Neither of these projects directly addressed an interface design.

In [18] an adaptive multimodal IVIS that filters input information based on both a priority scheme and the given situation is proposed. Tests showed a reduction in driver's workload and also more than $85 \%$ of participants were satisfied with this kind of system. 
2.3. HMI Design Principles. The design of a proper HMI interface for the purpose of ensuring a good user experience and road safety has been extensively studied, and as a result several guidelines and principles have been put forward. This section provides some background on the principles most widely used by developers and designers when defining an interface. These concepts are relevant for our purpose, since their baseline will be used when proposing adjusted principles for adaptive interfaces in vehicles. The majority of these principles are based on accumulative experience and heuristic experiments obtained through user feedbacks. Therefore, the results are closely related to human nature and how the mind processes information, as well as cultural and physiological factors.

In regard to road safety, the European Statement of Principles on Human Machine Interface (ESoP) has proposed a set of recommendations or principles in the areas of design, installation, information presentation, and system behaviour of in-vehicle information and communication systems [19]. From this set of recommendations, those with most relevance to HMI design are as follows:

(i) The system should not give rise to potentially hazardous behaviours and should prevent the distraction of the driver.

(ii) The information should be displayed in a uniform manner and be consistent among available interfaces.

(iii) Relevant information should be clearly displayed and the user should be able to detect and understand it with a few glances.

(iv) Priority should be assigned to the information so that may be displayed as rapidly as possible. Furthermore, it should be organized in such a way as to avoid the presentation of simultaneous messages and be displayed in a sufficient amount of time. Similarly, auditory messages should not mask high priority messages or warnings.

(v) The manual-visual action function should not be interrupted, although exceptionally it may be cancelled automatically or by the user when a certain amount of time has passed; for example, the driver should be able to enter half of a phone number, continue driving for some time and then go back to finish writing the phone number.

(vi) It is recommended that information unrelated to primary driving tasks should not be displayed while the vehicle is in motion. Moreover, the information should not be restored immediately when the vehicle is stopped, but only after a certain amount of time.

As regards the improvement of user experience and usability of the system, two notable research works in the area are the Eight Golden Rules regarding design interaction proposed by Schneiderman [20] and the 10 heuristic usability principles defined by Nielsen as an extension of the Golden Rules [21].

Schneiderman proposed the set of 8 rules derived from experience and heuristic experiments; these rules are recommended in order to achieve a well-designed interface.
The most significant design concepts contained therein are: consistency in the design style; always offer informative feedback about system status (loading, successful result, errors); provide easy reversal of actions to allow the user to undo or cancel started actions, and finally reduce short-term memory load so that the user is not obliged to remember information from one display or another.

In a similar research work, Nielsen performed experiments in order to detect usability problems by means of heuristic tests. In his results, he defined 10 basic principles, known as "heuristics," which can be used as guidelines for developing user interfaces. These principles were chiefly devoted to web design, but as HMI infotainment systems are quickly evolving and become increasingly more complex, this background provides a useful guide to design.

Some of these principles are similar to Schneiderman's Golden Rules, the most relevant of which are as follows: it presents an indicator about the system status in order to provide an understanding about what is happening with the system; the user should easily be able to cancel operations or return to previous views in case of mistakes or changes of opinion; the design must be consistent as regards colours, words, and icons in the application; the interface should display only the essential information in order to avoid overcrowded screens that may lead to confusion, and, finally, shortcuts should be accessible for executing the most common actions.

In another research, Gruhn sets out a set of concepts that may also be regarded as a design reference. He differentiates four main notions that should be taken into account in every design: Contrast of relevant things among the rest (emphasis); repetition of visual elements, alignment and interconnection between them, and proximity differentiation of linked or unrelated elements [22]. This research also points out the relevant importance of so-called "Situation Awareness," by which the user must always be conscious of the purpose of the current interface and how to access other contexts and have an overall idea about what will happen when an interaction is started.

\section{Materials and Methods}

3.1. Procedure. Based on previous studies, a set of HMI design principles for adaptive interfaces were compiled and then applied for modifying and existing HMI design that could be embedded into a Head Unit. It is true that a set of recommendations does not guarantee the creation of an ideal design, but provides an approach towards the enhancement of current designs when working with adaptive interfaces. The implementation was carried out in a real vehicle, since the purpose of this study is to evaluate the performance and user acceptance in real driving scenarios. The adaptive system was created using as baseline a commercial infotainment system belonging to the automotive manufacturer SEAT (Media System Plus 2015 Interface). This approach enables us to evaluate the real effect of the dynamic nature of the system in a commercially available developed interface.

HMI changes were determined by an indicator that estimates the driving complexity based on 5 variables. These 
TABLE 1: Participant profile.

\begin{tabular}{lccccc}
\hline Participants & Gender & Age & Driving frequency & Driving style & Appreciation by technology \\
\hline 15 & 10 males, 5 females & $28 \pm 4$ & 12 daily, 3 half-months & 11 average, 3 sportive & High (15/15) \\
\hline
\end{tabular}

variables exert a significant influence on driving complexity: road type, vehicle speed, wheel angle, traffic, and day/night state. Neither environmental conditions nor profile information was used to predict driving complexity, since all the trips were conducted in similar conditions (partly sunny day on a dry road), and the system was built to be as generic as possible in terms of subjective appreciation of diverse profiles.

The driving complexity predictor was built using statistical data collected from real driving scenarios. These data is processed, and after applying data mining techniques and machine learning methods a model was obtained. The HMI platform loads the created model and through the monitoring of vehicle's variables the driving complexity is computed at a sample time of $200 \mathrm{~ms}$.

A framework based on Open Services Gateway Initiative (OSGI) was used for creating the adaptive HMI. Through this framework, a server replicates available functionalities of the infotainment system. For the programming of the graphical interface, the modelling is implemented using JavaFx, a software platform for creating desktop applications and Rich Internet Applications (RIAs).

In this OSGI framework, a service is responsible for monitoring the output of the predictive model and notify each context when the current driving complexity changes. Since the HMI platform consists of a set of independent services (contexts), each one of these contexts is in charge of making proper interface changes according to the current driving complexity value. As previously stated, the goal is to ease the driver interaction with the infotainment system in very demanding scenarios and to offer a better user experience for low-demanding cases.

The adaptive design was implemented over the most common application context used while driving: Media (location and reproduction of storage songs); radio (tuning a radio station and saving stations as favourites); navigation (guidance-related task, finding location, and presentation of guidance manoeuvres); Vehicle Status (information about vehicle variables and current trip), and, finally, a Picture Viewer (browsing of pictures), which requires larger glance times and therefore is a more hazardous application to use when driving.

Once the system had been designed and embedded in the vehicle, an acceptance test was executed by selecting 15 participants ( 10 male, 5 female) who were asked to evaluate the system after using it on a previously defined route. According to Nielsen and Landauer [23], 5 participants are sufficient to detect $75 \%$ of usability problems, but since this test is also designed to evaluate user acceptance, the number of selected participants was increased to 15 . The "ThinkAloud" method, recommended in [24], was also incorporated in the test. This method is recommended to be implemented during the initial stages of design while concepts are still being formulated.

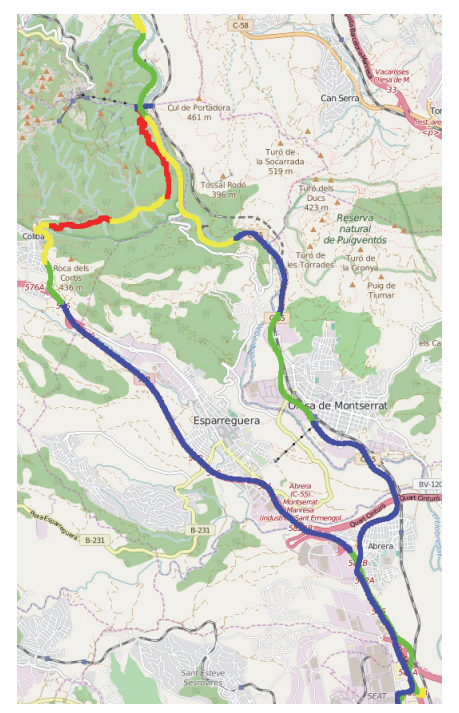

FIGURE 1: Road used in test and complexity segments.

Objective measures were not considered since this study was mainly oriented to evaluate users' perception and acceptance. The evaluation of a system design is generally given by users' subjective assessments and it is difficult to calculate as a set of objective values. With respect to workload, many objective parameters (physiological and driving performance when executing primary and secondary tasks) can be used. However, on the basis of de Waard et al's studies [25], who asserted that no one is able to provide a better judgement on the workload experienced than the subjects themselves, we prefer not to include those metrics in the test.

Participants were selected in order to obtain a homogeneous sample in terms of age, driving experience (more than $30,000 \mathrm{~km}$ driven), driving periodicity and how much they liked the technology, as shown in Table 1. Participation in the test was voluntary and informed written consent was taken from every participant.

The route driven in the test consisted of both complex and easy driving segments, including different road types such as motorways, secondary roads and tertiary roads with curves. All the participants characterized the route with the same perceived complexity and encountered similar levels of traffic. The route had a total length of $38 \mathrm{~km}$ and was travelled in an average time of 70 minutes. Figure 1 shows the route followed and the average complexity predicted by the estimator in each segment; colours represent the complexity: very-low (blue), low (green), high (yellow), very-high (red).

During the trip, the observer asked the participant to execute some actions on the HMI. These interactions were requested because in common driving scenarios the user may be unlikely to perform many actions while complexity was 


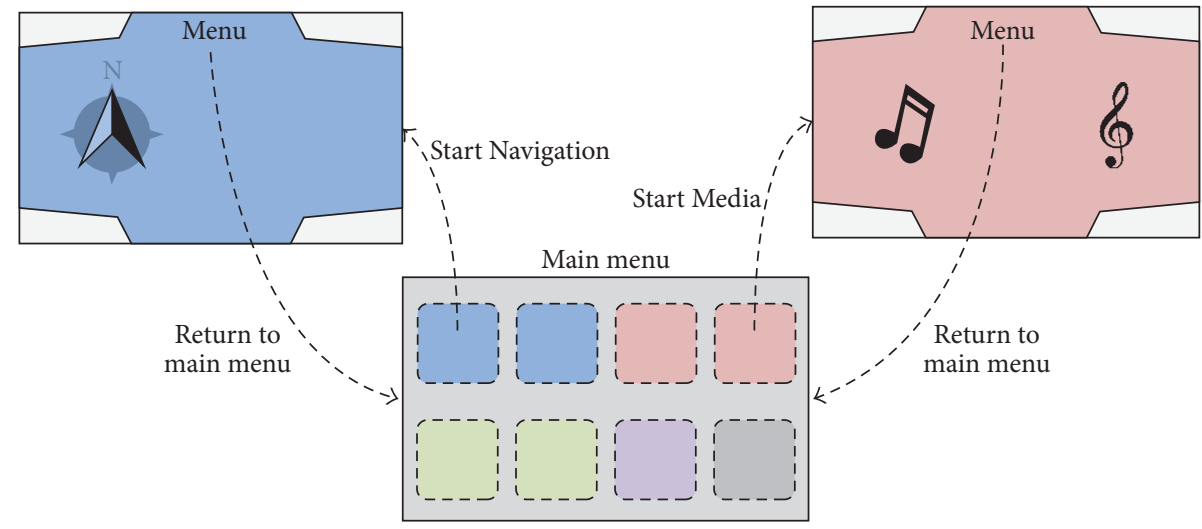

FIgURE 2: Baseline HMI used (main menu interaction).

high. All participants were able to observe all the changes in contexts as well as the adaptive nature of the system.

On completion of the trip, the participants were asked to fill out a questionnaire in order to validate three main concepts: evaluation of route driven in order to establish a consistency of route scenario perception; evaluation of the system as regards road safety benefits; and overall acceptance of this kind of system to be used while driving.

3.2. HMI Tested Design. The proposed HMI system adapts the interface according to four levels of driving complexity. Thus, as the level of complexity increases, the aim of the system is to ease driver interaction and thereby assure better road safety. A commonly employed and very useful strategy in adaptive systems is the implementation of a scheduler that delays the presentation of information depending on road conditions. However, this current study does not take the scheduler into account, because this is a field that has been studied extensively.

The baseline Head Unit has a main menu that provides the user with easy access to diverse applications. Each application has an accessible button to facilitate return to the main menu as it can be seen in Figure 2; most of the contexts have also a button in each corner associated with an action. The adaptive changes are defined independently in each context, but comply with the important principle of consistency between changes so that each context is adapted to the same degree. Also, an indicator of current driving complexity, represented by icon showing a iconographic face, was present in upper right part of each display to inform the driver about expected changes. This approach is aimed at increasing the driver's level of situational awareness (SA) and give information about the expected behaviour of the system.

The adaptive nature proposed is characterized by the implementation of two major design alterations; restrictions, focused on restricting some functionalities in complex scenarios; and appearance, focused on the reorganization and changes in how the information is displayed. Greater and lesser design alterations are implemented in order to evaluate user acceptance to different changes in this type of system.

Restriction alterations are applied during complex driving scenarios and on functionalities that are not commonly used or require a higher level of attention. To this end, functionalities are first classified according to three parameters: role inside the application; relevance to the user, and interaction complexity. Interaction complexity is objectively measurable and can be analysed with variables as glance time or the number of clicks required to complete the task. For example, some functionalities involving browsing through lists or writing messages using a keyword should be characterized as being of higher demand than functionalities such as clicking a "next" button to change a track or a song.

The classification of functionalities must be carefully planned, since the impact on usability and user experience will be directly linked to whatever restrictions are applied. Some nonrelevant functionalities suitable for restriction in complex scenarios are the search for a particular track on an album, the selection of POIs in the navigation, or the saving of new presets as favourites on the radio.

The alteration in appearance facilitates interaction with certain commonly used, low-complexity functionalities inside each application. The appearance of the design in high complex scenarios is more focused on usability and providing easy access to information rather than the look or aesthetics of it. Some of the incorporated changes could highlight the relevance of commonly used functions such as "next track," "play," "pause," or "stop-start." Other modifications could be made to hide nonrelevant information; for example, as regards navigation, areas outside the current route could be hidden in order to make map reading easier.

Design proposals for each context can be summarized as follows:

(i) Main Menu. Applications are presented in a grid format except in very-low-complexity cases (e.g., vehicle stopped) when the grid is changed by a carousel format. This design is oriented to increase user experience. A grid facilitates access to the applications since icons are distributed statically. On the other hand, a carousel is attractive but has a very dynamic arrangement in which applications are constantly changing location each time the carousel rotates. As a consequence, the time needed to identify applications is expected to be greater in carousels than in grid structures. 


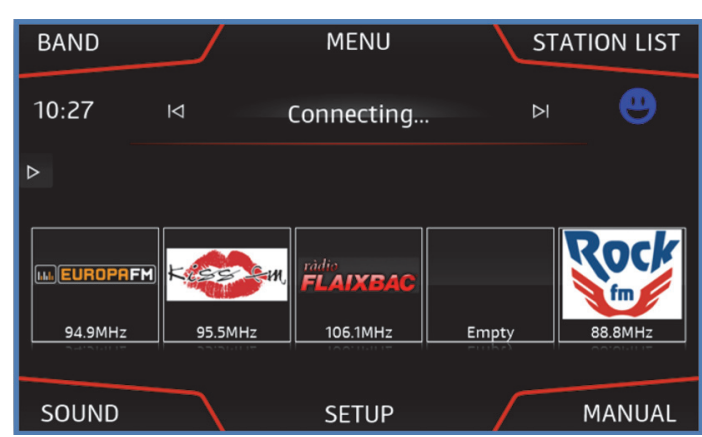

(a)

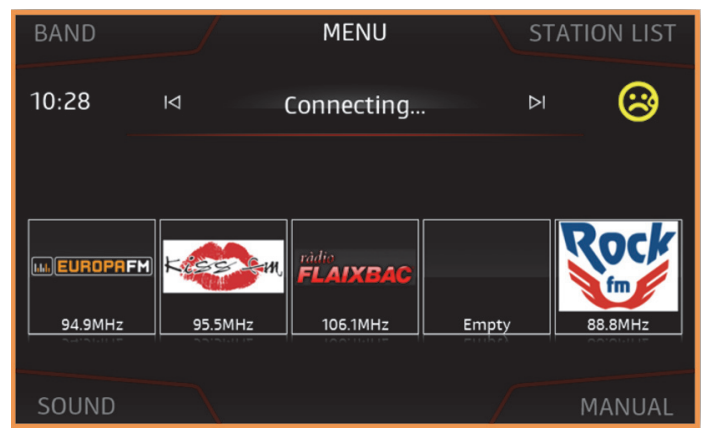

(c)

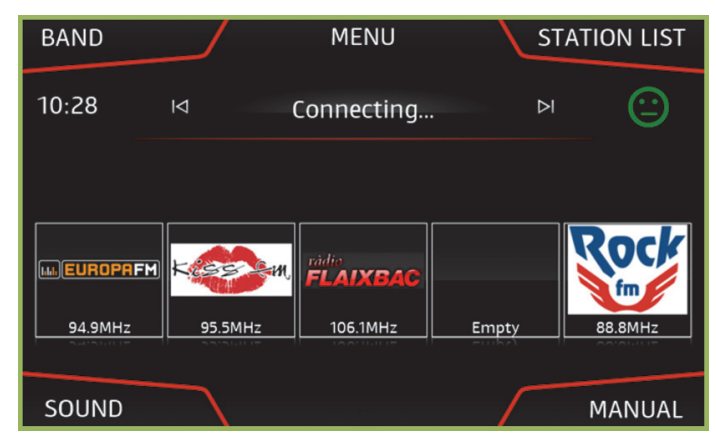

(b)

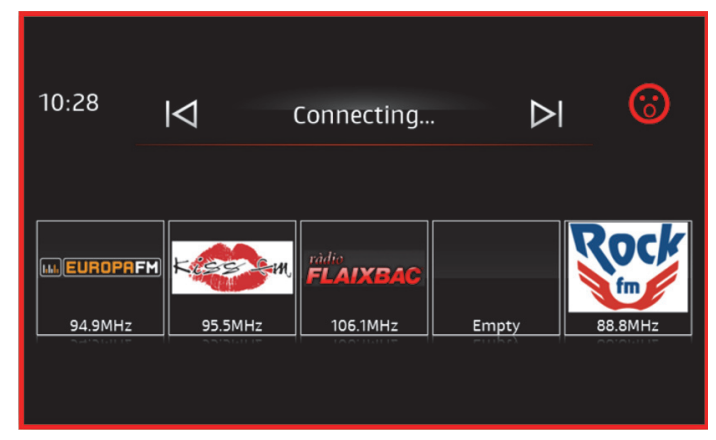

(d)

Figure 3: Radio context changes: very-low (a), low (b), high (c), and very-high (d).

(ii) Radio. In low-complexity scenarios (baseline) all functionalities are available; in second complexity level a panel indicating secondary navigation information is removed. In the third complexity level, actions associated with finding of station in lists and configuration of favourite presets is restricted. The interaction with lists requires a greater glance time towards the screen and therefore a reduction in the attention to the primary driving tasks is expected. Finally, in the last complexity level it is only allowed to change between predefined presets or next-previous stations. In this level, corner buttons are hidden to provide a more simplistic screen and evaluate participants opinions in this regard. The hiding of buttons could be considered a strong and intrusive alteration since functionalities available in the context are being removed from the screen. The purpose of this change was to compare user's perceptions under this approach and another in which buttons are deactivated by changing only their opacity contrast. Additionally, the size of the next/previous buttons is increased, an increment in a button size is recommended since the user is able to press the button faster, on the counterpart an increment in size lose certain design styles. Figure 3 shows the radio context for all driving complexity levels from very-low to very-high.

(iii) Media. When the complexity increases some functionalities are restricted (disabling top-right and hidden bottom-center buttons); sizes of "next," "previous," and "play-pause" buttons are increased; the deployable album selection is collapsed (fold-up), and some details about the album are hidden. These changes are made in order to reduce interface complexity and at the same time maintaining a proper level of user experience. When the driver is in a complex scenario some details regarding album title or release year are not imperative.

The right corner button gives access to a screen where a list of available songs is presented. The list can be ordered and filtered by song name, album, genre or author. If driving complexity level increases when the user is in this screen, the functionality is not immediately restricted, but instead delayed until the user complete the action and return to the Media main context. This approach is followed to avoid user experience problems; so that if users are already engaged in a functionality it is not blocked until the desired task is completed (we could name it as adequate timing principle). Figure 4(a) gives an example of a change in the restriction and appearance of Media from level very-low to level very-high.

(iv) Gallery. This application presents a viewer of images. The context design is very simple since it only contains two buttons for changing the image to the previous or next one. The design is not modified depending on the driving complexity due to its simplicity, but instead the application is completely removed from the main menu when the complexity reaches to high. If the user is interacting with the application at the moment of the change, the application is kept open 


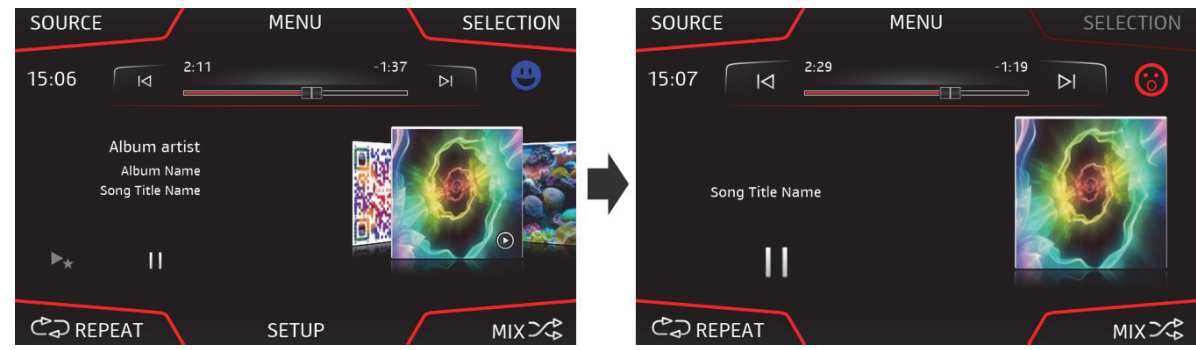

(a) Media context

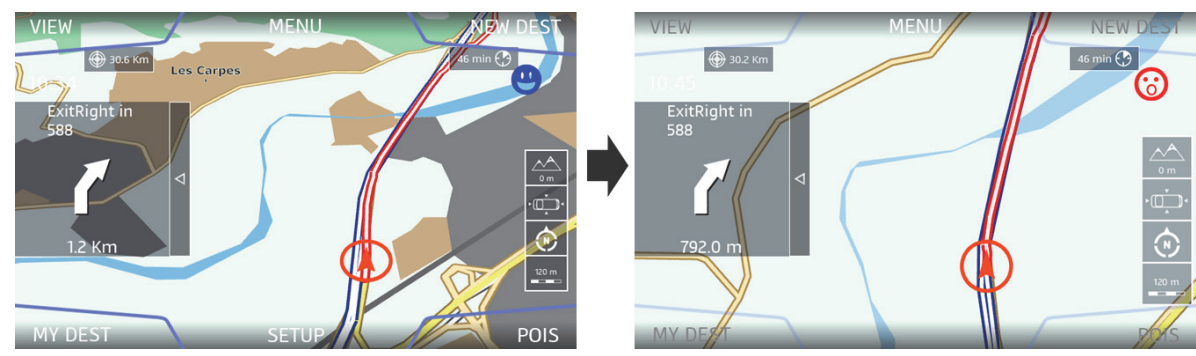

(b) Navigation context

FIGURE 4: Example of adaptations from very-low-complexity to very-high complexity.

until the user closes it. This approach follows the previously commented adequate timing principle and is oriented to keep a proper level of user experience.

(v) Navi. The navigation context is very dynamic and complex since it constantly renders a map that sometimes can contain numerous graphic elements. The baseline design contains a button in each corner which allows: setting a New Destination, changing the view, selecting preconfigured destinations, and selecting POIs. Two additional panels are presented in each side of the screen showing (1) navigation manoeuvres and (2) configuration of view parameters as zoom and orientation.

When the complexity increases to the second level, the button "New Destination" is blocked since for its use, typing in a keyboard is mandatory. For the third level (high), two other buttons in the corner are blocked: "POIs" which is useful for finding places by category, and "View" that allows changing the camera angle. Finally, in the last complexity level, all buttons are blocked (disabled) and the size of the side panels is increased.

A map contains numerous elements and areas representing different information. While driving, not all this information is mandatory for understanding the guidance and therefore maps' appearance is also modified depending on the complexity. In higher complexity levels (above or equal to high driving complexity), some areas, labels and roads are set invisible as: industrial areas, park areas, building areas, town labels with population lower than 50.000 inhabitants, tertiary roads, pedestrian roads and POIs. Figure 4(b) gives an example of a change in the restriction and appearance of Media from level verylow to level very-high.

\section{Results}

All the participants characterized the route with the same perceived complexity, which enabled us to ensure a homogeneous driving scenario in the tests. As regards acceptance of the system, Table 2 shows some aspects of what participants thought when evaluating the use of this type of system; the bottom row may be regarded as a summary of the results reflecting how much the participants liked the system. In general, this constitutes a high level of acceptance, even though some functionalities were highly modified and affected the common expectations of users when interacting with the interface. One may also see how most of the changes were detected by drivers and had no negative impact on user experience.

Table 3 shows the results of the questionnaire on road safety. These results show that participants tend to have a high appreciation that this type of system would help prevent accidents and increase road safety. Participants were also asked about the inclusion of new functionalities and applications in low-complexity scenarios, rather than limiting their interaction completely while driving, showing a positive feedbacks towards these additions.

\section{Discussion of Results}

Table 2 presented a summary of different subjective metrics evaluated in relation to the participants' acceptance levels. With regard to the "detection of changes in the system," the average value obtained was high, indicating that the participants were generally aware of system changes and 
TABLE 2: Evaluation of acceptance of the system.

\begin{tabular}{|c|c|c|c|c|}
\hline Issue regarding acceptance & Average & St. Dev. & Min & $\operatorname{Max}$ \\
\hline $\begin{array}{l}\text { Detection of changes in the system } \\
\text { (From } 1 \text { (none) to } 4 \text { (the majority)) }\end{array}$ & 3,40 & 0,74 & 2,00 & 4,00 \\
\hline $\begin{array}{l}\text { Interference of adaptive system on expected use } \\
\text { (From } 1 \text { (none) to } 4 \text { (much)) }\end{array}$ & 2,47 & 1,36 & 1,00 & 4,00 \\
\hline $\begin{array}{l}\text { Inconvenience arising from changes } \\
\text { (From } 1 \text { (none) to } 4 \text { (much)) }\end{array}$ & 1,53 & 0,74 & 1,00 & 3,00 \\
\hline $\begin{array}{l}\text { Getting used to this system after time } \\
\text { (From } 1 \text { (none) to } 4 \text { (much)) }\end{array}$ & 3,33 & 0,90 & 1,00 & 4,00 \\
\hline $\begin{array}{l}\text { Like having this system in his vehicle } \\
\text { (From } 1 \text { (none) to } 4 \text { (much)) }\end{array}$ & 3,40 & 0,91 & 1,00 & 4,00 \\
\hline
\end{tabular}

TABle 3: Perceived road safety.

\begin{tabular}{|c|c|c|c|c|}
\hline Issue regarding safety & Average & St. Dev. & Min & $\operatorname{Max}$ \\
\hline $\begin{array}{l}\text { Perceived impact of the system on road safety } \\
\text { (From } 1 \text { (negative) to } 4 \text { (positive)) }\end{array}$ & 3,73 & 0,46 & 3,00 & 4,00 \\
\hline $\begin{array}{l}\text { Opinion on integration of new functionalities in low-demand scenarios } \\
\text { (From } 1 \text { (nonadequate) to } 4 \text { (adequate)) }\end{array}$ & 3,53 & 0,64 & 2,00 & 4,00 \\
\hline $\begin{array}{l}\text { Opinion on restriction of functionalities in high-demand scenarios } \\
\text { (From } 1 \text { (nonadequate) to } 4 \text { (adequate)) }\end{array}$ & 3,73 & 0,59 & 2,00 & 4,00 \\
\hline
\end{tabular}

therefore a comparison of perceived effects is adequate to be performed.

Another metric compared was the "interference of the adaptive system on expected use," for which the average value reported was low but the high standard deviation is an indicator of low consistency among participants' responses, thereby limiting possible conclusions. With respect to the "inconvenience arising from the changes," the average value is low and is a possible indicator of small confusion experienced by participants when interacting with the system. Discrepancies in results may be given as a result of participants using the system for their first time as well as their prior knowledge of the original version of the system.

The last two metrics evaluated in Table 2, "getting used to this system after time" and "like having this system in his vehicle" show favourable results in regard to the integration of adaptive system in vehicles. For both cases, values obtained where higher than 3 and standard deviation values were not significant.

Overall, test results show a positive feedback of participant towards the inclusion of adaptive interfaces in vehicles. The design principles applied have shown to provide a good user experience as well as an increase in perceived road safety. These principles must be clearly aimed to understand user preferences in terms of the functionalities that are most frequently used. Furthermore, one of the main premises of such adaptation is the introduction of HMI changes without having a high impact on the ultimate purpose of the application.

Table 3 exposes participant's feedbacks with regard to road safety; the question "perceived impact of the system on road safety" is valuable for understanding user's perception of safety. Most participants reported the adaptive system offered benefits to road safety. This metric is relevant as any increase in road safety perception is expected to be associated with a higher level of user experience, given that users feel more confident and less stressed.

The last two questions presented in Table 3 are related to participant's opinion about the integration of new functionalities in low-demanding driving scenarios or restricting functionalities in high-demanding driving scenarios. In both cases, participants reported that they liked the idea of changing the number of dynamically displayed features. Results were positive and with a high level of consistency (observed standard deviation less than 0.64).

Participants were also asked to give opinions about the system during the test. A relevant opinion expressed by participants concerned the need to ensure a smooth transition process of adaptation when the road complexity changes. Moreover, changes must be stable over time and only be activated if the driving complexity is the same over a certain period of time; this would obviate highly dynamic interfaces that could affect user experience. One aim of the design is that regular users should not be aware of most of the restrictions, since in conditions of high driving complexity the frequent use of limited functionalities is not expected.

The importance of providing an accessible indicator showing the current driving complexity was also detected. This enables drivers to understand and remember what restrictions are being adapted. In the test it was found that, although the participants did not know in advance what the HMI changes were, they did not feel confused when the restriction was activated. The proposal for a future test is to evaluate the impact of the system when it is used on a regular basis in drivers' own vehicles, where driving behaviour is deemed to be more natural. 
With regard to the adaptation of more commonly used features, very positive feedbacks were received. All the participants thought that the increase in button size would help to reduce the glance time when performing a very common action such as changing tracks or radio stations.

Also, it emerged that most participants would prefer unavailable or restricted functionalities to be completely hidden rather than changing the contrast, since the opacity of a feature is not always understood as an unavailable feature. Nevertheless, this is a controversial point and mixed opinions are expressed about the benefits of each alternative.

Based on available design principles and participant's feedbacks, an adapted set of recommendations for adaptive interfaces can be proposed. As a starting point, this set of principles is suggested to be classified into 5 main groups: consistency between designs and interfaces used, selection of the appropriate modality to present information and allow users to interact with the system, adequate time to launch notifications and adapt the HMI, ensure a proper level of Situation Awareness and cope with a good representation of information that can be easily understood.

\section{Conclusions}

Due to the increasing amount of functionalities and services that are being added in driving scenarios, it is necessary to create a system capable of managing the flow of information. In this regard, a suitable adaptation of the interface under certain driving complexity scenarios may ease the interaction with applications.

This study deals with the evaluation of user acceptance of an adaptive HMI system that depends on driving complexity; as a result, a positive feedback was received from participants regarding the usability of the system. This test enabled us to define a strategy to be followed that is based on some of the design principles evaluated herein. The main benefits of the system in terms of road safety will become evident once drivers are accustomed to the behaviour of the system and understand that in certain scenarios complex functionalities may be restricted.

The proposed system makes smooth interface changes while maintaining a consistent design and facilitating interaction with the most used actions. This proposal could be an alternative to current systems that only consider as possible scenarios: driving or stopped. Likewise, the participants exposed an adequate benefit of this type of driving systems in regard to road safety.

As a future support to this type of system, a series of tests is proposed in order to obtain measurable values for comparing the benefits in distraction times; for example, a responsetime test employing mechanisms such as the Peripheral Detection Task (PDT) or an unawareness detection-time by means of Eye Tracking monitoring.

In addition, statistical data comparing the long-term benefits of various adaptive versus nonadaptive systems may provide fairly conclusive results. For this analysis, it is proposed to include more than one type of infotainment system and to apply on these the set of recommendations given.
Other considerations that must be taken into account in the strategy for a commercial implementation of the system are inclusion of the driver profile as a metric for personalizing the interface as regards user preferences and control of the functionalities that should be changed to a greater or lesser extent. A further relevant point is the detection of a passenger as copilot, since in general complicated tasks would be carried out by the passenger, although it is possible that such copilots may not know how to interact with the system or may have some impairment, and in this case the strategy may not fulfil the aims of the system for which it is designed.

\section{Competing Interests}

The authors declare that there is no conflict of interests regarding the publication of this paper.

\section{Acknowledgments}

This study is undertaken as part of an Engineering Doctorate project. This work is supported by the SEAT, S. A. Automotive Company, the Universitat Politècnica de Catalunya, and the Generalitat de Catalunya. The authors wish to acknowledge the contributions of the SEAT engineers who collaborated in the design of the project as well as the people who participated in the tests.

\section{References}

[1] A. P. de Miranda and J. S. Germaine, "New direction and trends for vehicle entertainment systems," SAE Technical Paper 201236-0518, SAE International, 2012.

[2] D. Shinar, M. Meir, and I. Ben-Shoham, "How automatic is manual gear shifting?" Human Factors, vol. 40, no. 4, pp. 647654, 1998.

[3] Road Safety Performance Index, "RANKING EU PROGRESS. 10th road safety performance index report," Tech. Rep., 2016.

[4] National Center for Statistics and Analysis, "Traffic safety facts," Report no. DOTHS812293, National Highway Traffic Safety Administration, Washington, DC, USA, 2016.

[5] National Center for Statistics and Analysis, "Distracted driving 2014," Traffic Safety Facts Research Note. Report No. DOT HS 812 260, National Highway Traffic Safety Administration, Washington, DC, USA, 2016.

[6] B.-M. Swanson, "Distracted Driving causes nearly 40\%, automotive fleet," 2000, 20-21.

[7] IRU (International Road Transport Union), "A scientific Study 'ETAC' European truck accident causation, executive summary and recommendations," Tech. Rep., IRU (International Road Transport Union), Brussels, Brussels, 2007, https://www.iru.org/ sites/default/files/2016-01/en-2007-etac-study.pdf.

[8] L. Jin, H. Xian, Q. Niu, and J. Bie, "Research on safety evaluation model for in-vehicle secondary task driving," Accident Analysis \& Prevention, vol. 81, pp. 243-250, 2015.

[9] European Commission, "Annual accident report 2015," Tech. Rep., European Commission, Brussels, Belgium, 2015.

[10] J. Melorose, R. Perroy, S. Careas et al., “Temporal fluctuations in driving demand: the effect of traffic complexity on subjective 
measures of workload and driving performance," Transportation Research Part F: Traffic Psychology and Behaviour, vol. 22, pp. 207-217, 2014.

[11] Institute for Road Safety Research, "SWOV Fact sheet (The influence of weather on road safety)," Tech. Rep., Institute for Road Safety, Leidschendam, The Netherlands, 2012.

[12] R. Lancaster and R. Ward, "The contribution of individual factors to driving behaviour: implications for managing workrelated road safety," Research Report 020, Health and Safety Authority, London, UK, 2002.

[13] C. D. Wickens, "Processing resources in attention, dual task performance, and workload assessment," in Varieties of Attention, R. Parasuraman and R. Davies, Eds., Academic Press, 1984.

[14] T. Brown, D. Marshall, J. Moeckli, and T. Smyser, "A Final Report of SAfety Vehicle(s) using adaptive interface technology (SAVE-IT) program: task 14a evaluation," Tech. Rep., 2007.

[15] A. Amditis, L. Andreone, A. Polychronopoulos, and J. Engström, "Design and development of an adaptive integrated drivervehicle interface: overview of the AIDE project," in Proceedings of the 16th IFAC Conference, vol. 38, no. 1, pp. 103-108, Prague, Czech Republic, July 2005.

[16] C. Baber, "Generic intelligent driver support: a comprehensive report of GIDS," Applied Ergonomics, vol. 25, no. 3, pp. 195-196, 1994.

[17] European Commission Project, "DRIVE II, application of a real-time intelligent aid for driving and navigation enhancement (ARIADNE)," Project V2004/14, European Commission, Geel, Belgium, 1993.

[18] H. S. Park and K.-H. Kim, "Adaptive multimodal in-vehicle information system for safe driving," ETRI Journal, vol. 37, no. 3, pp. 626-636, 2015.

[19] Commission of the European Communities, "ESoP-European Statement of Principles on human-machine interface," Tech. Rep., Commission of the European Communities, 2008.

[20] B. Shneiderman, Designing the User Interface: Strategies for Effective Human-Computer Interaction, Addison-Wesley, Boston, Mass, USA, 2010.

[21] J. Nielsen, "Usability heuristics," in Usability Engineering, chapter 5, pp. 115-163, Elsevier, 1993.

[22] P. Gruhn, "Human machine interface (HMI) design: the good, the bad, and the ugly (and what makes them so)," in Proceedings of the 66th Annual Instrumentation Symposium for the Process Industries, pp. 27-29, College Station, Tex, USA, January 2011.

[23] J. Nielsen and T. K. Landauer, "A mathematical model of the finding of usability problems," in Proceedings of the INTERACT '93 and CHI '93 Conference on Human Factors in Computing Systems (CHI '93), pp. 206-213, ACM, Amsterdam, The Netherlands, 1993.

[24] P. A. Green and J.-S. Park, "Evaluation of a navigation radio using the think-aloud method," International Journal of Vehicular Technology, vol. 2013, Article ID 705086, 12 pages, 2013.

[25] D. de Waard, A. Kruizinga, and K. A. Brookhuis, "The consequences of an increase in heavy goods vehicles for passenger car drivers' mental workload and behaviour: a simulator study," Accident Analysis and Prevention, vol. 40, no. 2, pp. 818-828, 2008. 


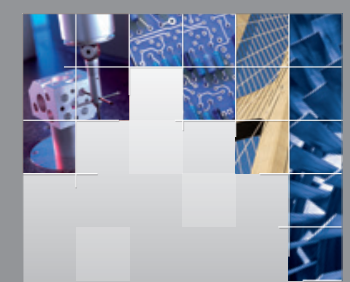

\section{Enfincering}
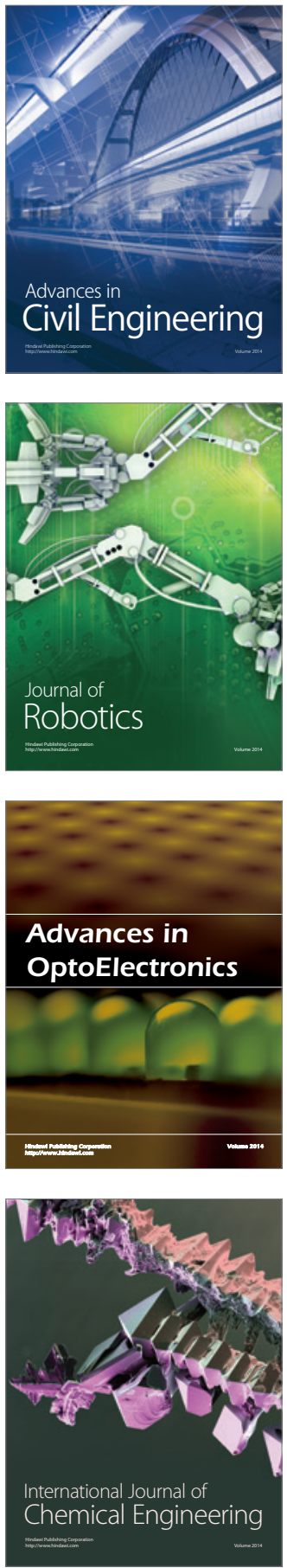

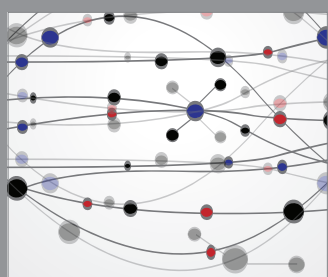

The Scientific World Journal

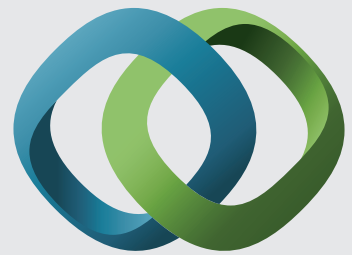

\section{Hindawi}

Submit your manuscripts at

https://www.hindawi.com
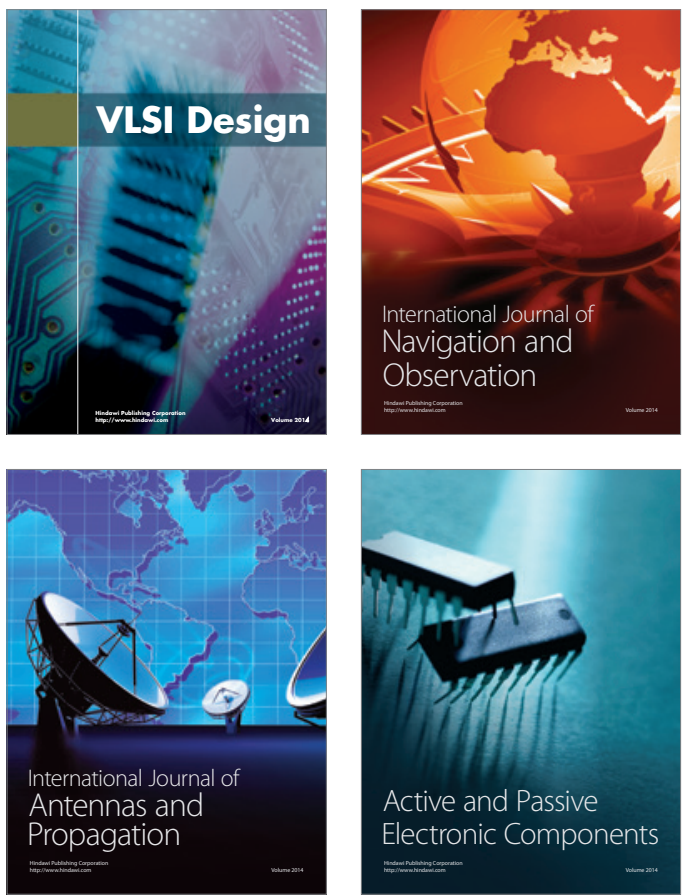
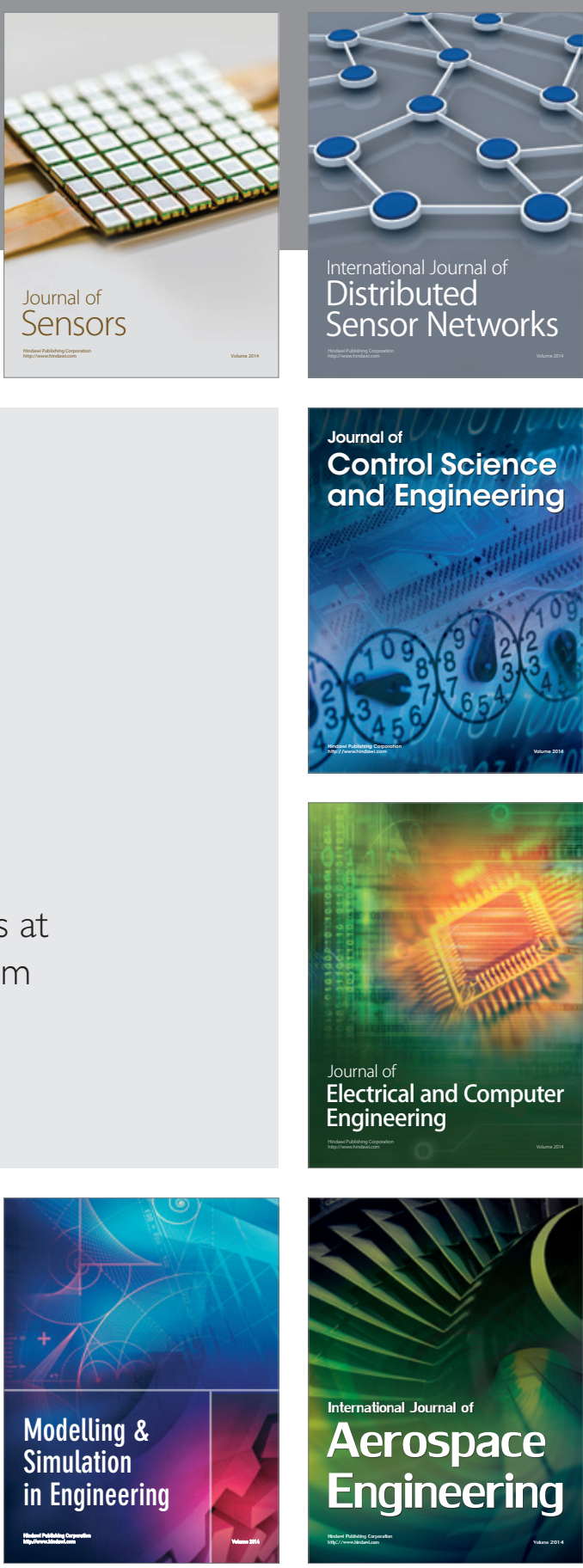

International Journal of

Distributed

Sensor Networks

$-$

Joumal of

Control Science

and Engineering
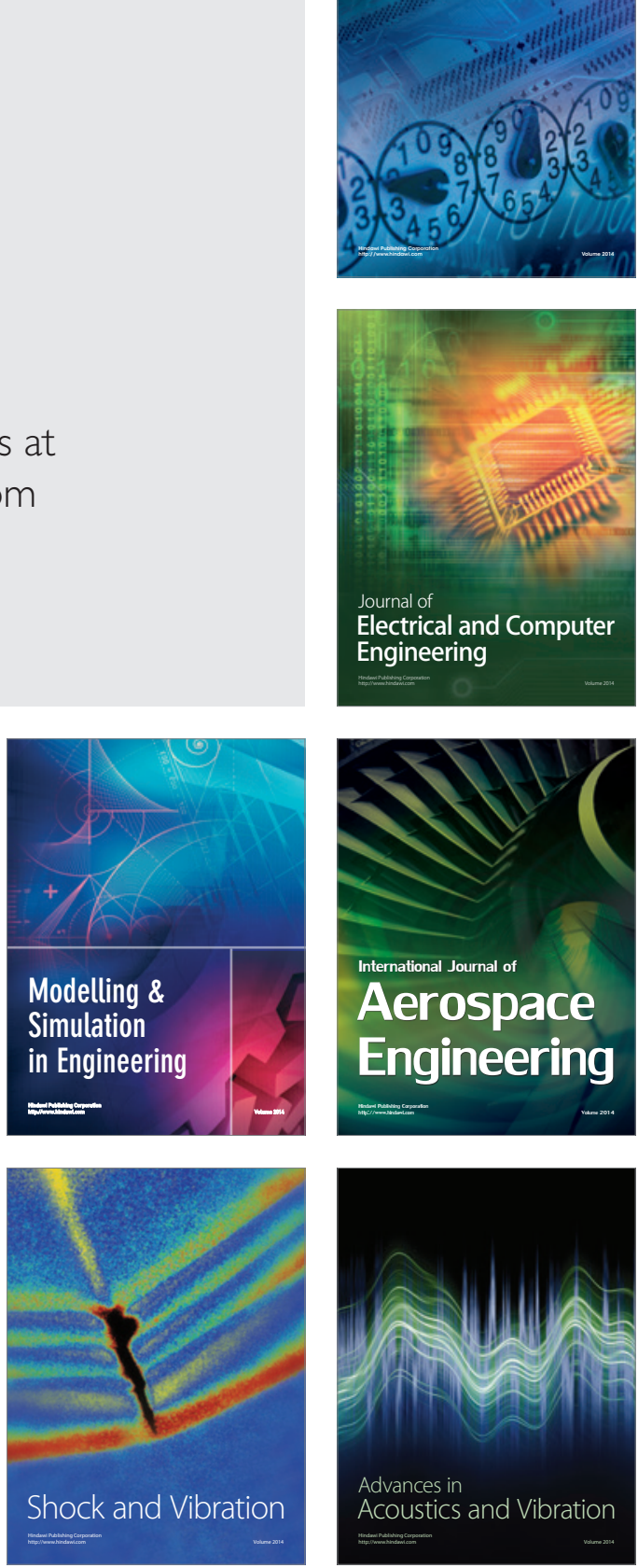